\title{
Capturing patient-reported area of knee pain: A concurrent validity study using digital technology in patients with patellofemoral pain
}

\author{
Mark Matthews $^{1,2}$, Michael S Rathleff ${ }^{3,4,5}$, Bill Vicenzino ${ }^{1}$, Shellie A Boudreau ${ }^{\text {Corresp. }} 5$ \\ ${ }^{1}$ School of Health and Rehabilitation Sciences, Sports Injuries Rehabilitation and Prevention for Health research unit, University of Queensland, Brisbane, \\ Australia \\ 2 Sports and Exercise Science Research Institute, School of Sport, Faculty of Life and Health Sciences, University of Ulster, Belfast, United Kingdom \\ 3 Research Unit for General Practice in Aalborg, Aalborg, Denmark \\ 4 Department of Occupational Therapy and Physiotherapy, Department of Clinical Medicine, Aalborg University, Aalborg, Denmark \\ ${ }^{5}$ Center for Neuroplasticity and Pain, Centre for Sensory Motor Interaction, Department of Health Science and Technology, Faculty of Medicine, Aalborg \\ University, Aalborg, Denmark \\ Corresponding Author: Shellie A Boudreau \\ Email address: sboudreau@hst.aau.dk
}

Background: Patellofemoral pain is often reported as a diffuse pain at the front of the knee during kneeloading activities. A patient's description of pain location and distribution is commonly drawn on paper by clinicians, which is difficult to quantify, report and compare within and between patients. One way of overcoming these potential limitations is to have the patient draw their pain regions using digital platforms, such as personal computer tablets.

Objective: To assess the validity of using computer tablets to acquire a patient's knee pain drawings as compared to paper based records in patients with patellofemoral pain.

Methods: Patients ( $\mathrm{N}=35)$ completed knee pain drawings on identical images (size and color) of the knee as displayed on paper and a computer tablet. Pain area expressed as pixel density, was calculated as a percentage of the total drawable area for paper and digital records. Bland-Altman plots, Intraclass correlation coefficient, Pearson's correlation coefficients and one-sample tests were used in data analysis.

Results: No significant difference in pain area was found between the paper and digital records of mapping pain area $(p=0.98)$, with the mean difference $=0.002 \%(95 \% \mathrm{Cl}-0.159-0.157 \%)$. A very high agreement in pain area between paper and digital pain drawings (intraclass correlation coefficient $=$ $0.966(95 \% \mathrm{Cl}$ 0.93-0.98, $F=28.834, d f=31, \mathrm{P}<0.001)$. A strong linear correlation $\left(\mathrm{R}^{2}=0.870\right)$ was found for pain area and the limits of agreement show less than $\pm 1 \%$ difference between paper and digital drawings.

Conclusion: Pain drawings as acquired using paper and computer tablet are equivalent in terms of total area of reported knee pain. The advantages of digital recording platforms, such as, quantification and reporting of pain area, could be realized in both research and clinical settings. 
1 Capturing patient-reported area of knee pain: A concurrent validity study using digital

2 technology in patients with patellofemoral pain

3 Matthews $\mathrm{M}^{1,2}$, Rathleff $\mathrm{MS}^{3,4,5}$, Vicenzino $\mathrm{B}^{1}$, Boudreau $\mathrm{SA}^{4}$

$4{ }^{1}$ The University of Queensland, School of Health and Rehabilitation Sciences, Sports Injuries

5 Rehabilitation and Prevention for Health research unit, CCRE Spine, Brisbane, Australia

$6{ }^{2}$ Sports and Exercise Sciences Research Institute, School of Sport, University of Ulster, Belfast,

7 United Kingdom

$8{ }^{3}$ Research Unit for General Practice in Aalborg, Department of Clinical Medicine, Denmark

$9{ }^{4}$ Center for Neuroplasticity and Pain (CNAP), Centre for Sensory Motor Interaction (SMI),

10 Department of Health Science and Technology, Faculty of Medicine, Aalborg University,

11 Denmark

$12{ }^{5}$ Department of Occupational Therapy and Physiotherapy, Department of Clinical Medicine,

13 Aalborg University Hospital

14 ADDRESS FOR CORRESPONDENCE

15 Corresponding Author: Shellie A. Boudreau,

16 Center for Sensory-Motor Interaction (SMIC)

17 Dept. Of Health Science and Technology,

18 Frederik Bajers Vej 7, DK 9220

19 Aalborg, Denmark

20 Phone: (+45) 99409829

21 Email: sboudreau@,hst.aau.dk 


\section{ABSTRACT}

23 Background:

24 Patellofemoral pain is often reported as a diffuse pain at the front of the knee during knee-loading 25 activities. A patient's description of pain location and distribution is commonly drawn on paper 26 by clinicians, which is difficult to quantify, report and compare within and between patients. One 27 way of overcoming these potential limitations is to have the patient draw their pain regions using 28 digital platforms, such as personal computer tablets.

29 Objective:

30 To assess the validity of using computer tablets to acquire a patient's knee pain drawings as 31 compared to paper based records in patients with patellofemoral pain.

32 Methods:

33 Patients $(\mathrm{N}=35)$ completed knee pain drawings on identical images (size and color) of the knee as 34 displayed on paper and a computer tablet. Pain area expressed as pixel density, was calculated as 35 a percentage of the total drawable area for paper and digital records. Bland-Altman plots, 36 Intraclass correlation coefficient, Pearson's correlation coefficients and one-sample tests were 37 used in data analysis.

38 Results:

39 No significant difference in pain area was found between the paper and digital records of 40 mapping pain area $(p=0.98)$, with the mean difference $=0.002 \%(95 \% \mathrm{CI}-0.159-0.157 \%)$. A very 41 high consistency in pain area between paper and digital pain drawings (intraclass correlation 42 coefficient $=0.966(95 \%$ CI $0.93-0.98, F=28.834, d f=31, \mathrm{P}<0.001)$. A strong linear correlation $43\left(\mathrm{R}^{2}=0.870\right)$ was found for pain area and the limits of agreement show less than $\pm 1 \%$ difference 44 between paper and digital drawings.

45 Conclusion:

46 Pain drawings as acquired using paper and computer tablet are equivalent in terms of total area of 47 reported knee pain. The advantages of digital recording platforms, such as, quantification and 48 reporting of pain area, could be realized in both research and clinical settings. 
49

50

51

52

53

54

55

56

57

58

59

60

61

62

63

64

65

66

67

68

69

70

71

72

73

74

75

76

77

\section{INTRODUCTION}

Pain drawings that capture area, location and distribution of pain can be used to aid diagnosis and track changes over time (e.g., after a course of treatment).[1-4] Pain drawings are commonly captured on paper-based body schemas, charts or sketched diagrams. [5-10] Despite studies reporting paper-based pain drawings to have good to very good inter-rater and intra-rater reliability, they present limitations for clinicians to easily quantify and compare pain areas within and between patients. [5-10] One way of overcoming these limitations is to have the patient draw the area and location of their pain on digital platforms, such as personal computer (PC) tablets. PC tablets offer considerable advantages to patients in health care settings. The advancements of touch-screen technology, such as those employed in smart-phones and hand-held PC tablets make it possible and easier to acquire, quantify, report and compare patient-completed pain drawings.

Recent studies have investigated pain drawings recorded on digital platforms and shown reliability commensurate with that of paper drawings [11 12] A study using high-resolution and contoured (3D) images of the knee found a high proportion of patients with patellofemoral pain (PFP) reported knee pain in mirrored locations and that the pain drawings were exceptionally symmetrical. [12] Interestingly, when the knee pain drawings were compared to duration of symptoms, those with a longer duration of symptoms appeared to draw patterns more of an ' $\mathrm{O}$ ' shape. Given that longer duration of PFP symptoms has been shown to be a prognostic indicator of a poor outcome, [13] capturing pain areas digitally will allow quantification and real time insight into the patient's condition, which may optimise patient management.

The aim of this study was to assess the concurrent validity of paper and digital pain drawings in patients with PFP. The hypothesis was that the area of knee pain in patients with PFP acquired using hand-held PC tablets (digital drawings) is equivalent to using pen and paper.

\section{METHODS}

This concurrent validity study investigated the agreement of pain area between patient completed paper and digital pain drawings. To minimise order and learning effect bias, the order of completing paper and digital pain drawings was randomised with approximately 1-2 minutes between drawings. The reporting of the study follows the Guidelines for Reporting Reliability and Agreement Studies [14]

78

79

80

81

82

83

84

85

86

87

88

89

90

91

\section{Participants}

A consecutive sample of 35 patients from a clinical trial [15] were recruited from the community of Aalborg, Denmark via public advertising or referred from Sports medicine clinics and general practitioners. A musculoskeletal physiotherapist with experience in managing patients with PFP screened participants for inclusion into the study. Inclusion criteria were (1) aged between 18-40 years with a history of non-traumatic anterior retro or peripatellar knee pain that was greater than six weeks duration (2) self-reported worst pain over the previous week equal to or greater than 3 out of 10 on a numerical pain scale $(0=$ no pain, $10=$ worst pain imaginable) (3) symptoms provoked by at least two of the following activities: squatting, running, stair ascending or descending or prolonged sitting were included. Individuals were excluded if they had any one of the following: concomitant injury or pathology of other knee structures (e.g. ligament, meniscal, tendon, iliotibial band, pes anserinus, fat pad), or a history of knee surgery, patellofemoral dislocation or subluxation, Osgood-Schlatter's disease, Siding-Larsen-Johanssen syndrome, a positive patellar apprehension test or evidence of knee joint effusion. The Ethics Committee in 
92 the North Denmark Region and the Danish Data Agency approved the study (N-20140022). All

93 participants were provided with verbal and written information about the procedures of the study,

94 and written informed consent was obtained prior to data collection.

95 Data collection

96 The participants were instructed by a second musculoskeletal physiotherapist to complete in a

97 randomised order a paper and digital pain drawing to the best of their abilities. The verbal

98 instruction given to the patients for both the paper and digital pain drawings was "Please use the

99 pen to draw on the paper/screen where you most often experience your knee pain". Digital pain

100 drawings were performed on a PC tablet (Samsung Galaxy Note 10.1, Android 4.1.2) that

101 displayed a 3D body schema of the lower torso (from the anterior superior iliac spine

102 prominences, and below, such that contours of the left and right legs and knees were clearly

103 visible) (Fig 1). Participants used a permanent red marker with a 1mm thick felt tip (Edding 400,

104 Germany) for the paper drawings and an S Pen ${ }^{\mathrm{TM}}$ that accompanied the tablet so as to control for

105 line thickness and to enable precise drawings. In order to enable a valid comparison (i.e., like

106 with like) three a-priori calibrations were performed: (i) the thickness of the line created by the S

$107 \mathrm{Pen}^{\mathrm{TM}}$ on the tablet was set to equal the thickness of the permanent red marker on the paper, (ii)

108 the S-Pen drawing on the digital device created red 'pixels' to indicate the patient's knee pain

109 location, area, and distribution and (iii) the image of the lower body schema as displayed on

110 paper was scaled to the same size as the lower body schema displayed on the computer tablet.

\section{FIGURE 1:}

112 Fig. 1 Unmarked 3D lower body leg schema on paper (A) and digital (B)

\section{Sample-size}

114 The hypothesis is that a hand held PC tablet is a comparable and equally valid method for

115 acquiring patient-completed pain drawings as compared to pen and paper records. Based on a

116 previous study, [16] it is hypothesized that the difference in pain area between these methods will

117 not be greater than $1 \%$. We used an equivalency sample-size calculation to determine the sample-

118 size required to show that there was no clinically relevant differences between pain area(s)

119 collected by paper versus pain area(s) collected using a computer tablet. The data used for the

120 sample-size calculation were based on means and standard deviations of two PFP groups

121 collected in a pilot study. Using a conservative correlation factor of 0.5 between drawings, an

122 equivalency limit of 6720 pixels and a SD of 4451 pixels at $5 \%$ significance and $95 \%$ power, it

123 was necessary to collect 35 paper-digital pairs of pain drawings.

124 Data management and analysis

125 To compare pain areas between paper and digital records, we calculated the pixel density of the

126 scanned paper and digitally acquired pain drawings. The pixel densities of the area of the pain

127 drawing on both paper and digital media were expressed as a percentage of the total area of a

128 blank body map of the lower body schema (i.e., a reference standard). Any pain areas that were

129 ambiguous in terms of the boundaries and extent of pain were excluded, such as cross-hatching

130 with unfilled areas, as shown in Figure 2(A-C).

131 Assessment of pain area for paper drawings

132 One investigator (MM) who was not involved in data collection and was blind to the computer

133 tablet records processed the paper records to determine pixel density of the paper based pain

134 recordings. This investigator scanned all the paper records for subsequent determination of pixel

135 density from the digital record. 
136 Paper drawings were scanned at 300 ppi, saved as a PDF file and imported into Adobe Photoshop

137 CC (Adobe Systems, 2015.1) for analysis. The pen selection function was used to trace a path of

138 the body schema and pain area to create a 'selected area' from which the pixel density was

139 calculated. First, a reference standard of pixel density for the paper version of the lower body

140 schema was created by scanning an unused paper version of the lower body schema. The total

141 pixel density was calculated three times and then averaged. The pain area for each participant's

142 paper record was traced and pixel density calculated.

143 Assessment of pain area of digital drawings

144 The Navigate Pain ${ }^{\mathrm{TM}}$ software that was preloaded on the PC tablet and automatically calculates

145 the red pixels associated with the pain drawings. The red pixels are also expressed relative to the

146 total pixel area (total drawable area) of the lower body schema. The percent and absolute number

147 of pixels were exported directly into an excel document for data analyses.

148 Data Analysis

149 One-sample t-tests were used to compare the difference in pixel density between paper and

150 computer table recordings of the patient's pain drawings. Intraclass correlation coefficient (ICC)

151 using absolute-agreement, two-way mixed model was used to determine the agreement between

152 paper and digital platforms. Pearson's correlation coefficients were used to express the degree of

153 linear association between the 2 methods [17]. Limits of agreements (LoAs), using Bland Altman

154 plots, were used to express the agreement between the paper and computer tablet methods. The

155 LoAs were presented as a range indicating the maximal potential difference between the 2

156 methods in 95\% of the ratings. All statistics were performed in IBM SPSS Statistics, version 24

157 (IBM Corp., Armonk, N.Y., USA) and $\alpha=0.05$ was used as level of significance.

158 RESULTS

159 Thirty-five participants were recruited into the study. Three participants were excluded, as they

160 did not follow the drawing instructions (Fig 2). One participant was excluded due to the use of

161 arrows in their paper drawing to indicate a pain area (Fig 2A). One participant was excluded due

162 to their paper drawing having incomplete circles with scribbled lines, leaving it unclear if it truly

163 represents their pain area (Fig 2B). One participant was excluded due to the ambiguous use of

164 zigzag lines in both the paper and digital to indicate pain area (Fig 2C). The remaining 32

165 participant drawings were analysed (Fig 3). Participants were predominantly female (78\%), mean

166 age of 24.5(5.6) years old, BMI of 23.7(3.4) and with average symptom duration of 69.7 (range

167 2-192) months. Twenty-five of the 32 participants reported and marked bilateral symptoms.

168 FIGURE 2

169 Fig. 2 The excluded pain drawings, which did not follow the drawing instructions such as the use

170 of arrows (A), circles and scribbled lines (B) and zig-zag lines (C).

171 FIGURE 3

172 Fig. 3 The variability of the thirty-two digital knee pain drawings, from patients diagnosed with

173 PFP, used to assess pain area between paper and digitally acquired drawings.

174 A very high agreement in pain area between paper and digital pain drawings as reflected by an

175 ICC of $0.966(95 \%$ CI $0.93-0.98, F=28.834, d f=31, \mathrm{P}<0.001)$. There was a strong linear

176 correlation in pain area between paper and digital pain drawings $(\mathrm{R}=0.93, \mathrm{P}<0.0001)$ (Figure 4), 
177 The drawings with the largest difference $(1.1 \%)$ and smallest difference $(0.05 \%)$ in pain area

178 between paper and digital pain drawings are depicted in Figure 5.

179 No significant difference was found between the two methods $(\mathrm{p}=0.98)$, with the mean difference 180 and $95 \%$ confidence interval (mean difference $=0.002 \%(95 \% \mathrm{CI}-0.159-0.157 \%)$ and the Bland-

181 Altman plot (Figure 6) all found to be lower than the pre-defined $\pm 1 \%$ equivalence margins.

182 FIGURE 4.

183 Fig. 4. A strong linear correlation in pain area between paper and digital pain drawings

184 FIGURE 5.

185 Fig. 5 Pain drawings associated with the smallest $(0.05 \%, \mathrm{~A})$ and largest $(1.1 \%$, B) differences in 186 pixel density between paper and digitally acquired pain drawings

187 FIGURE 6.

188 Fig. 6. Bland-altman plot showing the limit of agreement in pain area between paper and digital

189 pain drawings

\section{DISCUSSION}

191 This study found minimal differences in pain area recordings made on paper or a PC tablet.

192 Results indicate that any difference in area would likely be less than $\pm 1 \%$. These results support

193 the hypothesis and provide an important first step towards validation of digitally acquired pain

194 drawings for pain assessment in the knee.

195 Digital pain drawings offer the advantages of being easily acquired, quickly quantified and

196 interpreted to assist in clinical diagnosis and comparison over time. Previous studies have

197 investigated paper-based pain mapping to assist in clinical diagnosis of knee and shoulder pain.

198 [10 18 19] Participants were instructed to use small crosses ('X') [10] or symbols [18 19] to mark 199 out their pain location, type, distribution, and severity and several ' $X$ 's if pain was present in 200 more than one location. Once the drawing was made, a grid-like template was use for 201 categorizing anatomical zones of the knee. In its simplest form, placement of ' $X$ 's allow quick 202 reporting and the identification of a general location of the pain. However the utilisation of ' $X$ 's 203 to mark out pain area limits the accuracy of the patient expressing their pain distribution and 204 raises doubt on the diagnostic utility. By using a digital method in this current study, patients 205 were able to fully express their perceived pain location and distribution and not be restricted to 206 simple 'X's. Although not a focus of the present study, digital drawings could improve the 207 diagnostic accuracy of the pain drawings and be potentially useful and cost-effective as an 208 adjunct tool to quantify and interpret a patient's pain.

209 An unexpected observation in this study was the variability of the individual pain drawings in a 210 cohort with a homogenous diagnosis. Of the 32 participants, $23(72 \%)$ drew pain areas on both 211 knees with $12(38 \%)$ patients drawing pain in two or more locations in the same knee. This 212 observation has also been seen in previous studies. [5 10] Thompson et al [5] reported patients 213 indicating pain in 2 or 3 locations or 2 regions with several participants drawing 3 areas of local 214 pain. By drawing multiple areas, it could appear that participants are expressing diffuse 215 symptoms, multi-location or different pain types e.g. sharp or aching types of pain. The 216 variability of these drawings could also be a reflection of the heterogeneous nature of PFP. 217 Patellofemoral pain is an often persistent, multifactorial condition that is diagnosed by its clinical 218 presentation with exclusion of other conditions. [20] The simplified approach of this diagnosis 
219 could lead to the captured pain drawings expressing a variety of local nociceptive, peripheral and 220 central sensitization pain presentations. Whilst this current study compared the percentage of 221 pain area, the change in location of this area between drawings was not assessed. A change in 222 location between two pain drawings has yet to be assessed, even when considering the earliest 223 reliability studies. A change in location would of course be bodily-context dependent. With more 224 or less acceptable deviations in location change dependent on the pain being assessed such as the 225 knee or low back. The variability of the present drawings also warrants further consideration in 226 future studies, looking at the relationship between patient-perceived pain drawings, location and 227 diagnosis.

228 The level of anatomical detail displayed on the body schema used in the current study may have 229 contributed to the minimal difference obtained between paper and digital pain drawings. In a 230 pain-mapping study on a chronic neck pain cohort, results found high reproducibility between 231 paper and digital platforms as well as between simple body outlines and high-resolution 232 contoured body schemas. [11] However, a small fixed negative bias was identified with slightly 233 smaller drawings performed on paper than PC tablet, and pain areas were drawn slightly larger on 234 the less-detailed body outline in comparison to the high-resolution body schemas. [11] One 235 explanation for these findings could be the greater level of anatomic detail of the body schema 236 being more recognizable to the patient. When a patient is able to see important anatomical 237 landmarks, greater accuracy and precision of the pain drawings may occur.

238 A key consideration of this study is the verbal instructions given to the participants in the study. 239 The instructions given may have allowed some degree of ambiguity. This is evident by three of 240 the 35 excluded pain drawings. The pain drawings were excluded due to the amounts of 241 unmarked areas within circles and use of zigzag lines for shading in larger areas. As a 242 recommendation for future studies the instruction set should be of explicit clarity and possibly, 243 include a sample example of a correct and incorrect pain drawing. For example "Please draw on 244 the image that best represents the location and area of your pain. Please use solid lines or 245 completely filled in areas, leaving no clear spaces within the area".

246 A second consideration for this study was the method of acquiring the pain drawings. Clinicians 247 have traditionally completed pain drawings on body schemas, charts or sketched diagrams. [5-10] 248 However, considerations were identified in these studies that warranted attention. A comparison 249 study found that clinicians drew significantly smaller areas of pain when compared to the 250 patients' drawing, [9] suggesting observer bias and filtering of information by the clinician which 251 may not accurately represent the patient's report. In the current study, this consideration was 252 addressed by asking patients to complete the drawings. It is imperative future studies of pain 253 drawings, and indeed clinical utilization, ensure patient-completed pain drawings in guiding the 254 diagnosis. [9]

255 The result of this study opens possibilities on the benefits of using digital platforms in clinical 256 examination. By combining touch-screen technology with a high-detailed body schema, pain 257 drawings can be quickly quantified and interpreted to facilitate clinical decisions. In turn, 258 clinicians can easily monitor symptoms by comparing pain drawings within and between patients 259 over time. With continued development of software, new avenues could be created for research. 260 Future studies could explore and interpret pain drawings to enable identification of previously 261 unknown pain patterns. Identification of pain patterns could be particular pertinent in patients 262 with persistent and prevalent conditions such as knee or low back pain. Several studies on 263 patients with low back pain have used pain drawings to locate body regions where patients have 
264 experienced pain. [21 22] Results suggest pain drawings might help to understand the patient's 265 underlying mechanism of pain and improve treatment outcomes. [21 22]. The advantages offered 266 by digital recording platforms, such as automatic quantification and reporting of pain area, could 267 be realized in both clinical settings and research to improve healthcare.

268

269

270

271

272

273

274

275

276

277

\section{8}

279

280

281

282

283

284

285

286

287

288

289

290

291

292

293

294

295

296

297

298

299

300

301

302

303

304

305

306

307

\section{CONCLUSION}

This study found knee pain drawings acquired on digital and paper based platforms to be comparable in area. This study provides a first important step for testing of a digital interface that can facilitate the precise communication of patient-perceived knee pain area and location. The use of digital technology in health care, including digital platforms of patient-perceived pain drawing records, opens up many exciting possibilities in clinical and research settings.

\section{ACKNOWLEDGEMENTS}

Richard Spence for bulk extraction of the pixel density calculations from the Navigate Pain software and for technical support. Mette Bøgedal for screening and including the participants. Lukasz Winiarski for data collection

\section{REFERENCES:}

1. Margolis RB, Tait RC, Krause SJ. A rating system for use with patient pain drawings. Pain 1986;24(1):57-65.

2. Abbott JH, Foster M, Hamilton L, Ravenwood M, Tan N. Validity of pain drawings for predicting psychological status outcome in patients with recurrent or chronic low back pain. Journal of Manual \& Manipulative Therapy 2015;23(1):12-19.

3. Southerst D, Côté P, Stupar M, Stern P, Mior S. The Reliability of Body Pain Diagrams in the Quantitative Measurement of Pain Distribution and Location in Patients with Musculoskeletal Pain: A Systematic Review. Journal of Manipulative and Physiological Therapeutics 2013;36(7):450-59.

4. MacDowall A, Robinson Y, Skeppholm M, Olerud C. Pain drawings predict outcome of surgical treatment for degenerative disc disease in the cervical spine. Upsala Journal of Medical Sciences 2017:1-7.

5. Thompson LR, Boudreau R, Hannon MJ, Newman AB, Chu CR, Jansen M, Nevitt MC, Kwoh CK. The knee pain map: Reliability of a method to identify knee pain location and pattern. Arthritis and Rheumatism: Wiley Subscription Services, Inc., A Wiley Company, 2009:725-31.

6. Wood LRJ, Peat G, Thomas E, Duncan R. Knee osteoarthritis in community-dwelling older adults: are there characteristic patterns of pain location? Osteoarthritis and Cartilage: Elsevier, 2007:615-23.

7. Sengupta M, Zhang YQ, Niu JB, Guermazi A, Grigorian M, Gale D, Felson DT, Hunter DJ. High signal in knee osteophytes is not associated with knee pain. Osteoarthritis and Cartilage: Elsevier, 2006:413-17.

8. Creamer P, Lethbridge-Cejku M, Hochberg MC. Where does it hurt? Pain localization in osteoarthritis of the knee. Osteoarthritis and Cartilage: Elsevier, 1998:318-23.

9. Post WR, Fulkerson JP. Knee pain diagrams: Correlation with physical examination findings in patients with anterior knee pain. Arthroscopy J. Arthroscopic Relat. Surg., 1994:618-23.

10. Elson DW, Jones S, Caplan N, Stewart S, Gibson ASC, Kader DF. The photographic knee pain map: Locating knee pain with an instrument developed for diagnostic, communication and research purposes. The Knee: Elsevier B.V., 2010:1-7. 
308

309

310

311

312

313

314

315

316

317

318

319

320

321

322

323

324

325

326

327

328

329

330

331

332

333

334

335

336

337

338

339

340

341

342

343

11. Boudreau SA, Badsberg S, Christensen SW, Egsgaard LL. Digital Pain Drawings: Assessing Touch-Screen Technology and 3D Body Schemas. Clinical Journal of Pain 2016;32(2):139-45.

12. Boudreau SA, Kamavuako EN, Rathleff MS. Distribution and symmetrical patellofemoral pain patterns as revealed by high-resolution 3D body mapping: a cross-sectional study. BMC Musculoskeletal Disorders 2017;18(1):160.

13. Matthews M, Rathleff MS, Claus A, McPoil T, Nee R, Crossley K, Vicenzino B. Can we predict the outcome for people with patellofemoral pain? A systematic review on prognostic factors and treatment effect modifiers. British Journal of Sports Medicine 2016.

14. Kottner J, Audige L, Brorson S, Donner A, Gajewski BJ, Hrobjartsson A, Roberts C, Shoukri M, Streiner DL. Guidelines for Reporting Reliability and Agreement Studies (GRRAS) were proposed. International Journal of Nursing Studies 2011;48(6):661-71.

15. Matthews M, Rathleff MS, Claus A, McPoil T, Nee R, Crossley K, Kasza J, Paul S, Mellor R, Vicenzino B. The Foot Orthoses versus Hip eXercises (FOHX) trial for patellofemoral pain: a protocol for a randomized clinical trial to determine if foot mobility is associated with better outcomes from foot orthoses. J Foot Ankle Res 2017;10:5.

16. Boudreau SA, Badsberg S, Christensen SW, Egsgaard LL. Digital Pain Drawings: Assessing Touch-screen Technology and 3D Body Schemas. Clinical Journal of Pain, 2015.

17. Koo TK, Li MY. A Guideline of Selecting and Reporting Intraclass Correlation Coefficients for Reliability Research. Journal of Chiropractic Medicine 2016;15(2):155-63.

18. Bayam L, Ahmad MA, Naqui SZ, Chouhan A, Funk L. Pain mapping for common shoulder disorders. American Journal of Orthopedics (Belle Mead, N.J.) 2011;40(7):353-8.

19. Bayam L, Arumilli R, Horsley I, Bayam F, Herrington L, Funk L. Testing Shoulder Pain Mapping. Pain Medicine 2017.

20. Crossley KM, Stefanik JJ, Selfe J, Collins NJ, Davis IS, Powers CM, McConnell J, Vicenzino B, Bazett-Jones DM, Esculier J-F, Morrissey D, Callaghan MJ. 2016 Patellofemoral pain consensus statement from the 4th International Patellofemoral Pain Research Retreat, Manchester. Part 1: Terminology, definitions, clinical examination, natural history, patellofemoral osteoarthritis and patient-reported outcome measures. British Journal of Sports Medicine 2016;50(14):839-43.

21. Hullemann P, Keller T, Kabelitz M, Freynhagen R, Tolle T, Baron R. Pain Drawings Improve Subgrouping of Low Back Pain Patients. Pain Pract 2017;17(3):293-304.

22. Gerhardt A, Eich W, Janke S, Leisner S, Treede RD, Tesarz J. Chronic Widespread Back Pain is Distinct From Chronic Local Back Pain: Evidence From Quantitative Sensory Testing, Pain Drawings, and Psychometrics. Clinical Journal of Pain 2016;32(7):568-79. 


\section{Figure 1}

Unmarked 3D lower body leg schema on paper (A) and digital (B).
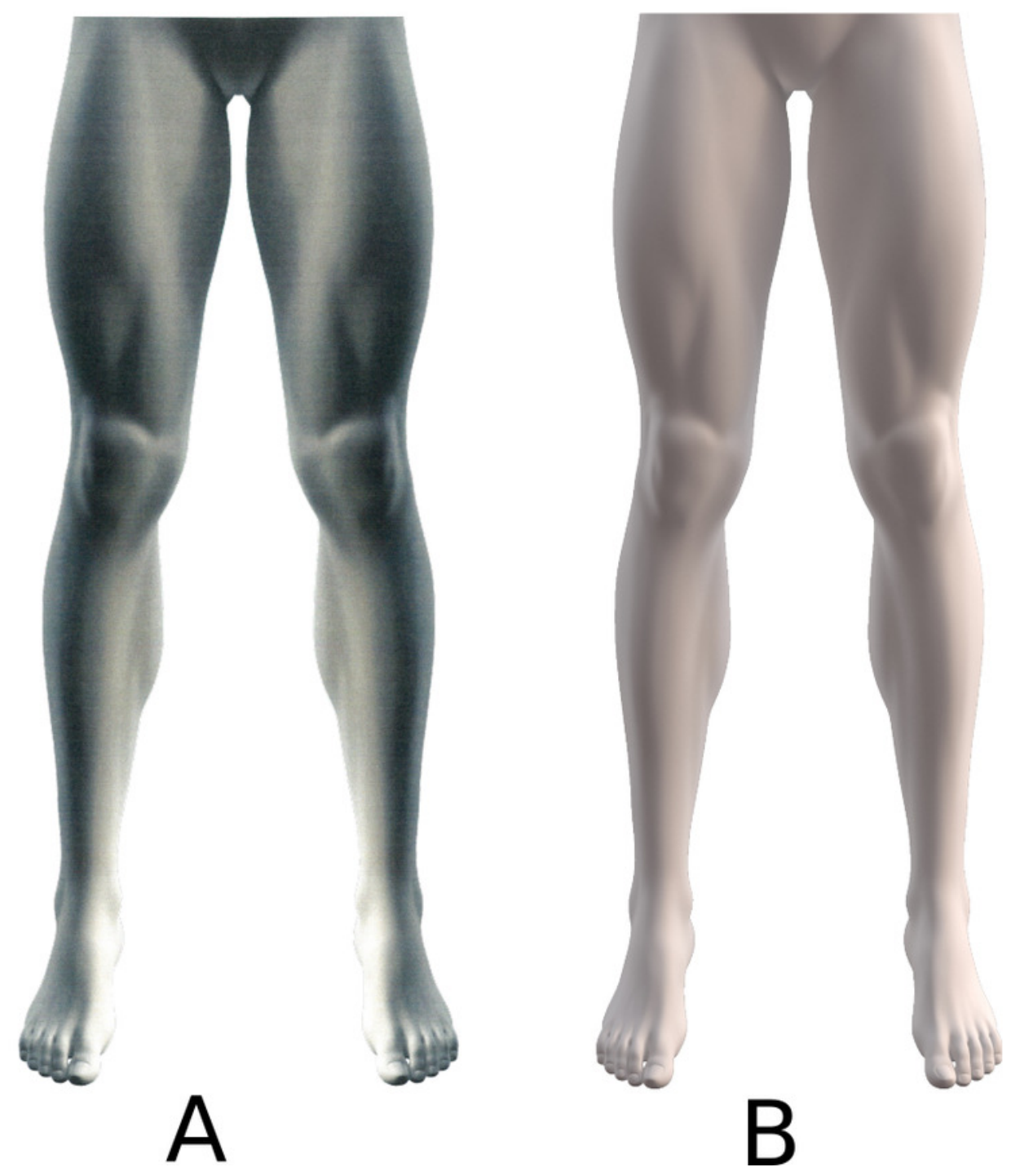
Figure 2

The excluded pain drawings, which did not follow the drawing instructions such as the use of arrows (A), circles and scribbled lines (B) and zig-zag lines (C). 


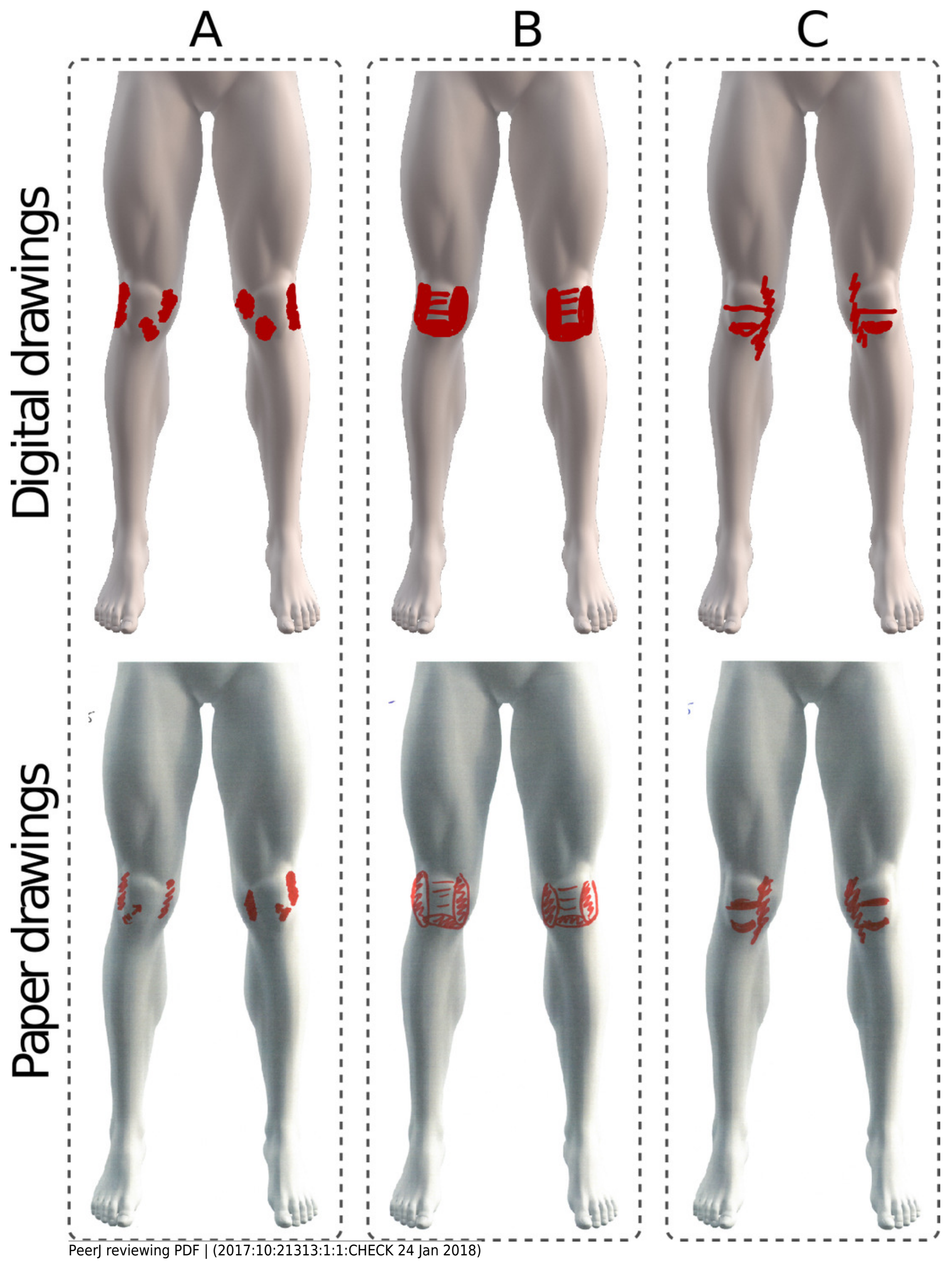


Figure 3

The variability of the thirty-two digital knee pain drawings, from patients diagnosed with PFP, used to assess pain area between paper and digitally acquired drawings. 

Figure 4

A strong linear correlation in pain area between paper and digital pain drawings

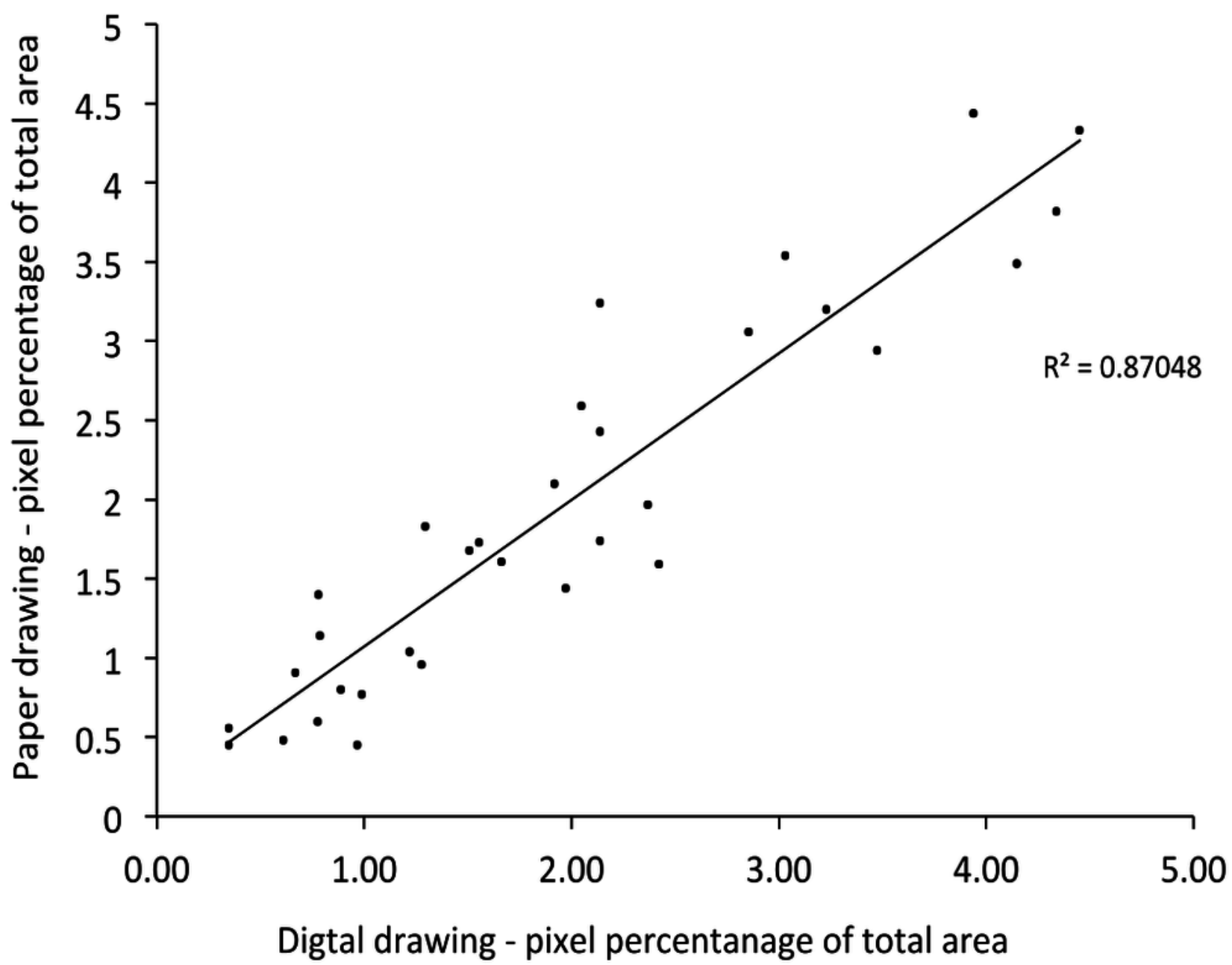




\section{Figure 5}

Pain drawings associated with the smallest $(0.05 \%, A)$ and largest $(1.1 \%, B)$ differences in pixel density between paper and digitally acquired pain drawings.

A

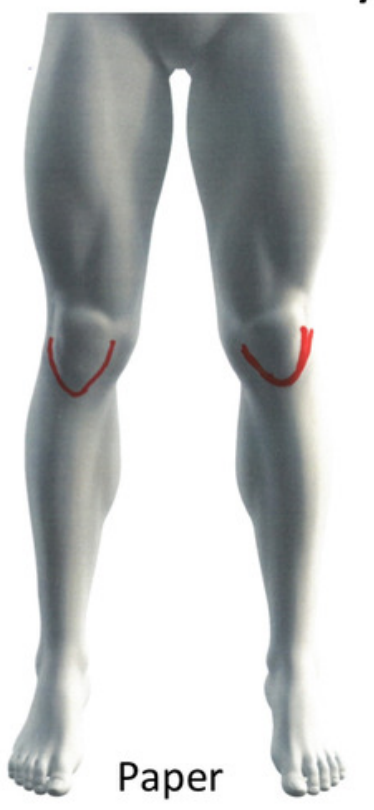

$A$

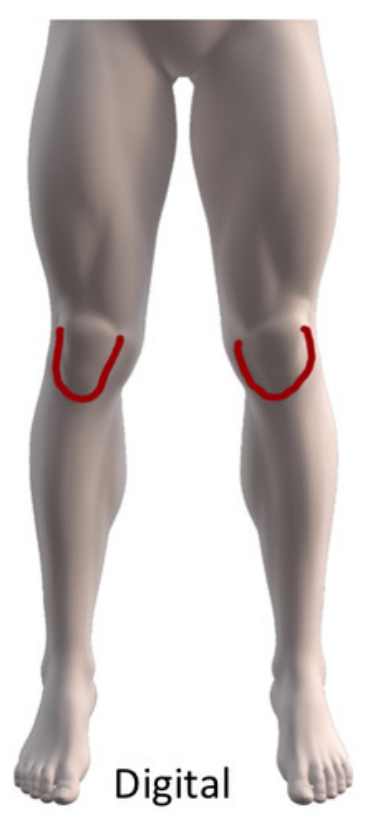

B

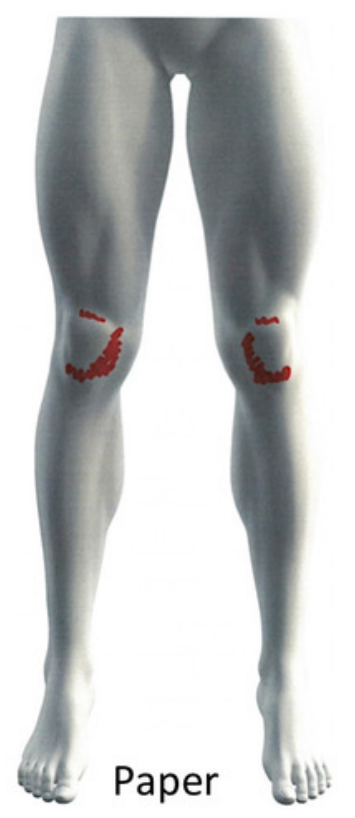

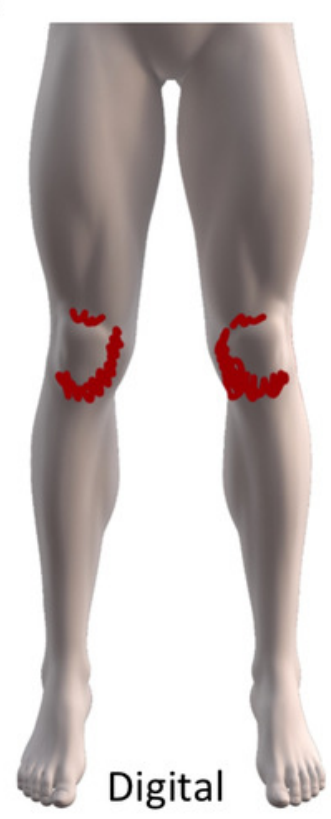


Figure 6

Bland-altman plot showing the limit of agreement in pain area between paper and digital pain drawings

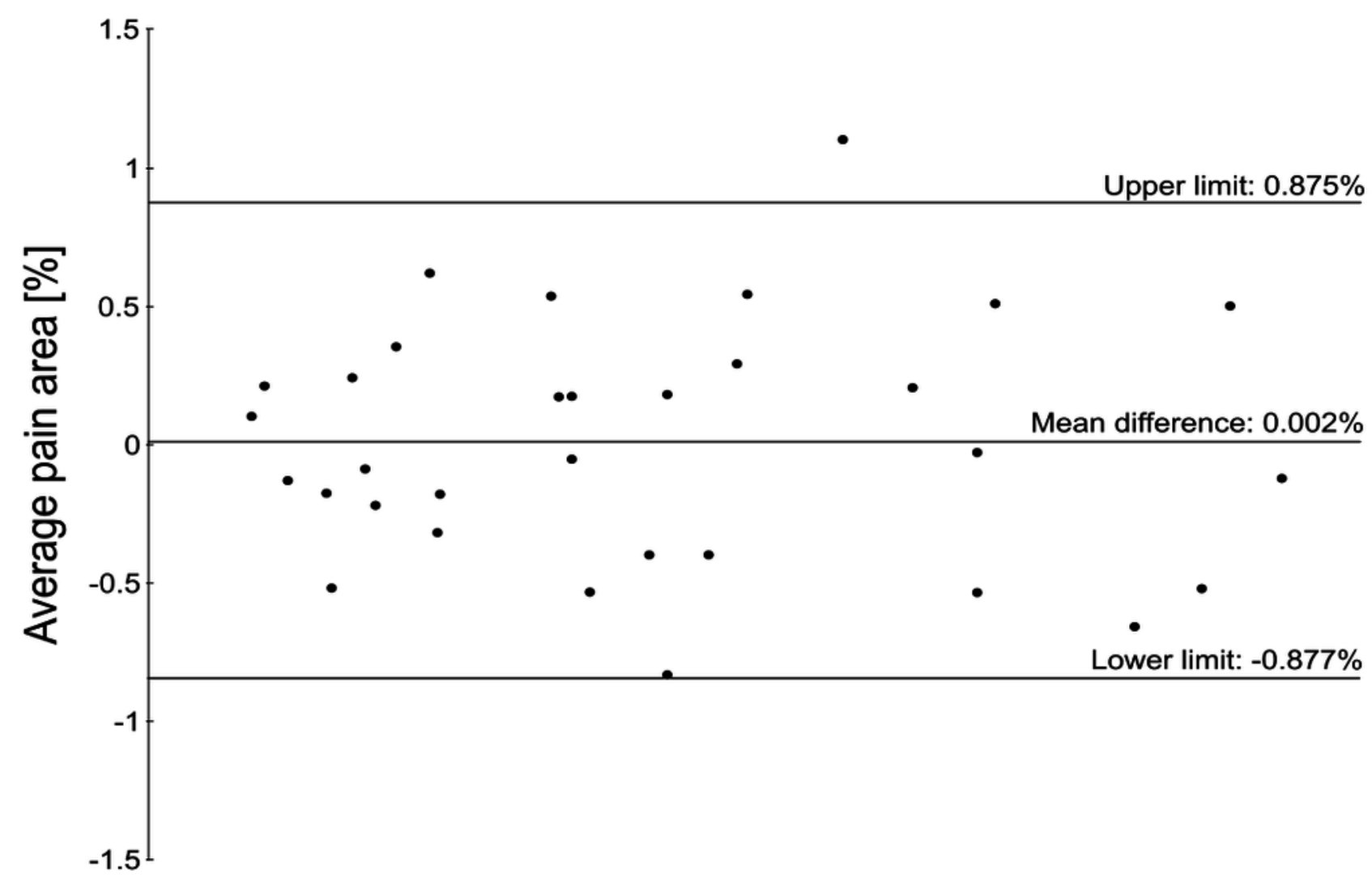

